\title{
Chicago Sociology
}





\section{Chicago Sociology \\ Jean-Michel Chapoulie}

Foreword by William Kornblum

Translated by Caroline Wazer

Columbia University Press

New York 


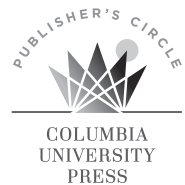

Columbia University Press gratefully acknowledges the generous support for this book provided by Publisher's Circle member Harriet Zuckerman.

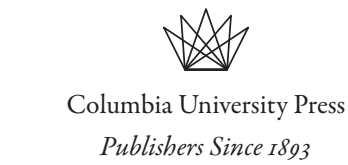

New York Chichester, West Sussex

cup.columbia.edu

First published in French as La tradition sociologique de Chicago, 1892-196I

by Jean-Michel Chapoulie

(C) 200 I Editions du Seuil

English translation copyright $\left({ }_{0} 2020\right.$ Columbia University Press

All rights reserved

Library of Congress Cataloging-in-Publication Data

Names: Chapoulie, Jean-Michel, author. | Wazer, Caroline, translator.

Title: Chicago sociology / Jean-Michel Chapoulie; foreword

by William Kornblum; translated by Caroline Wazer.

Other titles: Tradition sociologique de Chicago. English

Description: I Edition. | New York City : Columbia University Press, 2020. |

Includes bibliographical references and index.

Identifiers: LCCN 2019054948 | ISBN 9780231182508 (hardback) |

ISBN 9780231182515 (trade paperback) | ISBN 9780231544207 (ebook)

Subjects: LCSH: Chicago school of sociology.

Classification: LCC HM463.C4313 2020 | DDC 301.0977/311-dc23

LC record available at https://lccn.loc.gov/2019054948

Columbia University Press books are printed on permanent and durable acid-free paper.

Printed in the United States of America

Cover image: Magnum Photos $\odot$ Henri Cartier-Bresson, Chicago,

Illinois, USA, 1947

Cover design: Chang Jae Lee 